\title{
Encontro de Haemagogus (Conopostegus) leucocelaenus (Diptera: Culicidae), no Município de Porto Alegre, Estado do Rio Grande do Sul
}

\author{
Finding of Haemagogus (Conopostegus) leucocelaenus (Diptera: Culicidae), \\ in the municipality of Porto Alegre, State of Rio Grande do Sul
}

\author{
Almério de Castro Gomes ${ }^{1}$, Maria Amélia Nascimento Torres ${ }^{2}$, Lisiane Ferri ${ }^{3}$, \\ Felipe Rocha da Costa ${ }^{3}$ e Allan Martins da Silva ${ }^{4}$
}

\begin{abstract}
RESUMO
Em novembro de 2006, foi realizada uma investigação entomológica numa mata nativa do município de Porto Alegre, Estado do Rio Grande do Sul. O resultado foi a captura de várias espécies Culicidae, incluindo o primeiro registro da presença de Haemagogus leucocelaenus na localidade. Esta espécie é considerada vetora do vírus da febre amarela em alguns municípios do Estado, motivando esta comunicação para alertar sobre o potencial da área para circulação do agente etiológico desta doença.
\end{abstract}

Palavras-chaves: Município de Porto Alegre. Haemagogus leucocelaenus. Febre amarela silvestre. Inquérito entomológico.

\section{ABSTRACT}

In November 2006, an entomological investigation was carried out in a native forest in the municipality of Porto Alegre, State of Rio Grande do Sul. The result was the capture of several Culicidae species, including the first recording of the presence of Haemagogus leucocelaenus in this locality. This species is considered to be a vector for the yellow fever virus in some municipalities of this State, and this was the motivation for the present communication, in order to warn regarding the potential of this area for circulation of the etiological agent for this disease.

Key-words: Municipality of Porto Alegre. Haemagogus leucocelaenus. Wild yellow fever. Entomological survey.

0 vírus da febre amarela está distribuído numa vasta área do território brasileiro, mas a incidência da doença tem apresentado grande variabilidade ${ }^{3}$. 0 domínio dos casos é da Região Amazônica e fora dela a sua re-emergência passou a incluir, mais recentemente, os Estado da Bahia, Minas Gerais, São Paulo e Rio Grande do Sul' . Este último estado que era considerado, até alguns anos atrás, região indene foi incluído na área de transição ou de evidências de circulação viral $^{1}$. Isto se deu pelas epizootias em primatas não humanos, pois os últimos casos da doença foram registrados no ano de $1966^{2}$.

Em 2001, epizootias em macaco do gênero Alouatta emergiram no Rio Grande do $\mathrm{Sul}^{6}$, o que implicou no exame deste animal e mosquito Culicidae para comprovação da presença do vírus. Os municípios de Garruchos, Santo Antônio das Missões, situados a Noroeste e Jaguarí na parte central do estado, foram sede dos últimos eventos epizoóticos em macacos do gênero Alouatta ${ }^{6}$. No Município de Santo Antônio, o mosquito Haemagogus leucocelaenus foi encontrado com o vírus amarílico ${ }^{8}$. A partir deste encontro, a espécie ficou incriminada como responsável pela transmissão da febre amarela silvestre ${ }^{7}$.

A Cidade de Porto Alegre dista cerca de $650 \mathrm{~km}, 550 \mathrm{~km}$ e 400km das Cidades de Garruchos, Santo Antônio e Jaguari, respectivamente, É a área urbana do estado com maior densidade demográfica e, sobretudo, convivendo com Aedes aegypti. Por outro lado, pouco se sabe sobre a distribuição deste vetor no Rio Grande do Sul, a não ser pelas pesquisas que permitiram registrar a presença de Haemagogus leucocelaenus nos municípios já citados e nos de Três Passos, Soledade e Mata5 (Figura 1).

No dia 23 de novembro de 2006, foi realizada uma pesquisa exploratória numa mata nativa com 132,8 hectares, localizada em meio à área urbana no bairro Agronomia para conhecimento da riqueza de espécie Culicidae. Foi empregado o método da aspiração em abrigos naturais, a armadilha de Shannon e captura manual com puçá, com a duração de três horas, período da tarde até o crepúsculo vespertino. No vizinho Município de Viamão e

1. Faculdade de Saúde Pública, Universidade de São Paulo, São Paulo, SP. 2. Centro de Vigilância em Saúde, Secretaria Estadual de Saúde do Rio Grande do Sul, Porto Alegre, RS. 3. Profill Engenharia e Ambiente, Porto Alegre, RS. 4. Centro de Saúde Ambiental, Secretaria Estadual de Saúde do Paraná, Curitiba, PR.

Endereço para correspondência: Dr. Almério de Castro Gomes. FCS/USP. Av. Dr. Arnaldo 715, 01246-904 São Paulo, SP.

e-mail: agcastro@usp.br

Recebido em: 07/03/2007

Aceito em: 27/04/2007 


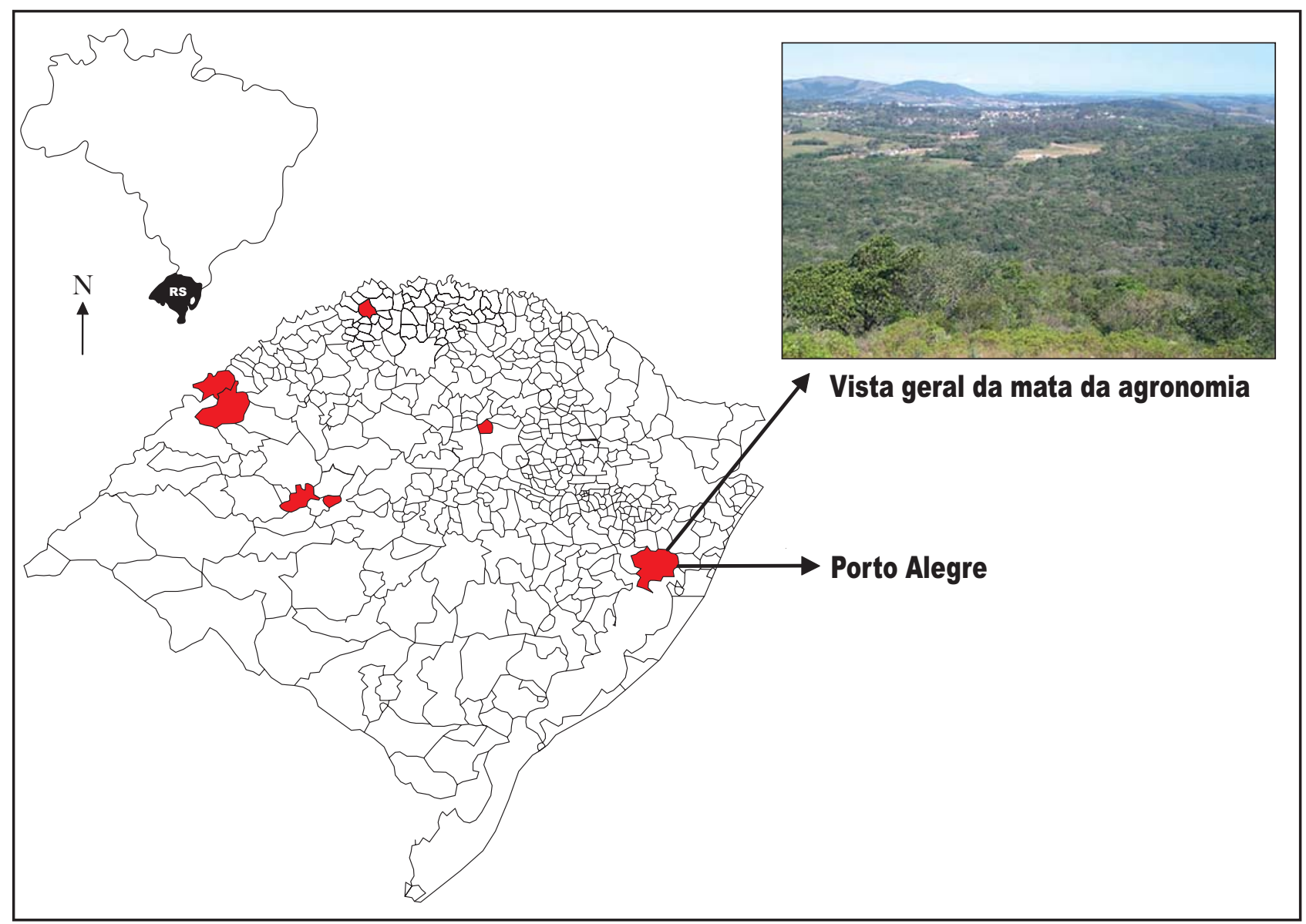

Figura 1 - Municípios do Rio Grande do Sul com Haemagogus leucocelaenus.

na zona sul de Porto Alegre, numa extensão de cerca de 40km, são encontrados macacos do gênero Alouatta. 0 resultado foi a captura de várias espécies de culicídeos e dentre essas o primeiro encontro de Haemagogus leucocelaenus no Município. A Figura 1 mostra a atual distribuição de Haemagogus leucocelaenus no estado, bem como a mata do bairro Agronomia em Porto Alegre, onde a espécie foi encontrada.

Diante da presença do vetor, de macacos Alouatta e das epizootias transcorridas recentemente no Estado do Rio Grande do Sul, justifica-se a divulgação do novo encontro de Haemagogus leucocelaenus, pelo fato de sua presença representar o principal elo para circulação do vírus da febre amarela na localidade. Como conseqüência, existe o risco potencial de envolvimento da população humana que reside ou trabalhe próximo à área.

Assim sendo, esta comunicação é um alerta para gestores do Sistema Único de Saúde (SUS) e, principalmente, para o Centro Estadual de Vigilância em Saúde da Secretaria de Saúde do Estado do Rio Grande do Sul (CEVS-SES). É necessário julgar a conveniência e oportunidade de incluir Porto Alegre no grupo de municípios do estado sob vigilância de epizootias produzidas pelo vírus da febre amarela. Também, seria oportuno monitorar a eventual passagem deste vírus à população humana, principalmente diante de epizootias em macacos que se elevam no país desde $1998^{7}$, pois Tauil ${ }^{4}$ ressaltou que o encontro da espécie em ambiente urbano merece uma atenção espacial.

Vale ainda ressaltar que nos inquéritos entomológicos realizados nos municípios riograndenses já citados, não foi encontrado
Haemagogus janthinomys, suspeitando-se de que sua distribuição geográfica não inclua o Estado do Rio Grande do Sul.

\section{REFERÊNCIAS}

1. Ministério da Saúde. Manual de vigilância de epizootias em primatas não humanos. Secretaria de Vigilância em Saúde; Brasília, 2005.

2. Nobre A, Antezana D, Tauil PL. Febre amarela e dengue no Brasil: epidemiologia e controle. Revista da Sociedade Brasileira de medicina Tropical 27 (supl III): 59-66, 1994.

3. Silveira AC. Febre amarela: aspectos epidemiológicos e de controle. Revista da Sociedade Brasileira de Medicina Tropical 31 (supl II) : 75-78, 1998.

4. Tauil PL. Haemagogus leucocelaenus em ambiente urbano. Revista da Sociedade Brasileira de Medicina Tropical 18:5-6, 1985.

5. Torres MAN, Almeida MAB, Santos E, Monteiro, HAO, Cardoso JC, Costa IA, Ferreira FB. Vigilância Entomológica da febre amarela silvestre no Rio Grande do Sul. Boletim Epidemiológico, Centro Estadual de Vigilância em Saúde, Secretaria da Saúde do Estado do Rio Grande do Sul 6: 6-7, 2004.

6. Torres MAN, Santos E, Almeida MAB, Cruz LL, Sperb AF. Vigilância da febre amarela silvestre no Rio Grande do Sul. Boletim Epidemiológico, Centro Estadual de Vigilância em Saúde, Secretaria Estadual da Saúde do Rio Grande do Sul 5: 1-8, 2003.

7. Vasconcelos PFC, Costa ZG, Rosa EST, Luna E, Rodrigues SG, Barros VLRS, Dias JP, Monteiro HAO, Oliva OFP, Vasconcelos HB, Oliveira RC, Sousa MRS, Silva JB, Cruz ACR, Martins EC, Ros MRS. Epidemic of jungle yellow fever in Brazil, implications of climatic alterations in disease spread. Journal of Medical Virology 65:598-604, 2001.

8. Vasconcelos PFC, Sperb AF, Monteiro HAO, Torres MAN, Sousa MRS, Vasconcelos HB, Mardini LBF, Rodrigues SG. isolations of yellow Fever virus from Haemagogus leucocelaenus in Rio Grande do Sul State, Brazil. Transaction of the Royal Society of Medicine and Hygiene 97: 60-62, 2003. 